\title{
EFECTO DEL TIPO GENÉTICO Y LA MADURACIÓN SOBRE LA RETENCIÓN DE AGUA EN CARNE DE TOROS CASTRADOS
}

\author{
EFFECT OF GENETIC TYPE AND AGING ON WATER HOLDING CAPACITY IN MEAT \\ FROM CASTRATED BULLS
}

\author{
Leal-Gutiérrez, J.D. ${ }^{\text {*; }}$ Jiménez-Robayo, L.M. ${ }^{\text {; }}$ Ariza, M. ${ }^{2}$; Manrique, C. ${ }^{2}$; López, J. ; \\ Martínez, C. ${ }^{2}$; Pinilla, Y. ${ }^{1}$; Ríos, M. ${ }^{2} ;$ Ortiz, Y. ${ }^{2}$ y Jiménez, A. ${ }^{4}$
}

\begin{abstract}
'Unidad de Genotipificación de Animales Domésticos (UGA). Departamento de Producción Animal. Facultad de Medicina Veterinaria y de Zootecnia. Universidad Nacional de Colombia. Bogotá. Colombia. *jdlealg@unal.edu.co

${ }^{2}$ Departamento de Producción Animal. Facultad de Medicina Veterinaria y de Zootecnia. Universidad Nacional de Colombia. Bogotá. Colombia.

${ }^{3}$ Instituto de Ciencia y Tecnología de Alimentos ICTA. Universidad Nacional de Colombia. Bogotá. Colombia. ${ }^{4}$ Asociación Colombiana de Criadores de Ganado Cebú. ASOCEBÚ. Colombia.
\end{abstract}

\section{Palabras clave adicionales}

Calidad cárnica. Jugosidad. Mermas. Pérdidas por cocinado. Razas criollas.

\section{RESUMEN}

La capacidad de retención de agua en crudo (CRAr) y en cocinado (CRAc) fue determinada en carne madurada a 7,14 y 21 días post mórtem. Los músculos Longissimus dorsi (LD) y Semitendinosus (ST) de 164 machos castrados provenientes del cruce de machos de las razas Simmental, Normanda, Blanco Orejinegro, Braunvieh, Guzerat, Brahman Blanco, Brahman Rojo, Limousin y Romosinuano con hembras Brahman blanco. Para el parámetro CRAr en LD se presentó significancia de los factores tipo genético y maduración y en ST del factor maduración. Para el parámetro de CRAc en LD fue significativo el factor tipo genético y para ST ninguno de los dos factores fue significativo. No existieron interacciones significativas de los dos factores evaluados. Lo ideal es someter a tiempos cortos de maduración ambos músculos y tener en cuenta el tipo genético al cual pertenece el individuo con el fin de minimizar las pérdidas de exudado.

\section{SUMMARY}

Water Holding Capacity (WHC) was established in raw meat ( $\mathrm{WHCr}$ ) through the press method and in cooked meat by cooking losses

\section{AdDitiONAL KEYWORDS}

Cooking losses. Creole breeds. Juiciness. Meat quality. Weight losses.

(WHCc). The beef was aged at 7, 14 and 21 days post mortem. The muscles Longissimus dorsi (LD) and Semitendinosus (ST) of 164 castrated males were used. These animals were F1 offsprings of Simmental, Normande, BON (Blanco Orejinegro), Braunvieh, Guzerat, White Brahman, Red Brahman, Limousin and Romosinuano bulls crossed with White Brahman females. In WHCr genetic type and ageing were significant factors in LD and ageing was significant in ST. Ideally, meat of ST and LD should be aged for short time post mortem and the genetic type can affect the water retention in LD.

\section{INTRODUCCIÓN}

En el trópico colombiano, los sistemas productivos ganaderos cuentan con un recurso genético muy importante, constituido por las denominadas razas criollas, que son el resultado de la multiplicación de ganado abandonado o perdido a partir de los cuales se crearon rebaños salvajes o cimarrones, cuya progresiva adaptación al medio, originó animales cada vez más resis- 


\section{LEAL-GUTIÉRREZ ET AL.}

tentes (Beteta, 1997). En este grupo de razas se encuentran el Romosinuano y el BON (Blanco Orejinegro) radicando su importancia en la variabilidad genética responsable de su adaptación. Sánchez et al. (2005) demostraron que estas dos razas poseen una mayor riqueza alélica cuando se compararon con otras poblaciones de ganado bovino criollo de Sudamérica. Cabe destacar que esta variabilidad genética es un gran potencial para la selección de individuos de genética superior (Renand et al., 2001), viéndose reflejado en la productividad del sistema (cantidad, calidad y bienestar), pero es indispensable realizar estudios que evalúen parámetros productivos de estas razas con el fin de establecer su potencialidad y contribuir al desarrollo del sector agropecuario (Anzola, 2005).

Las razas criollas han cumplido un proceso de adaptación de aproximadamente 500 años, lo que les ha proporcionado la habilidad de aprovechar forrajes de mala calidad, así como la adquisición de características como docilidad, habilidad materna, precocidad, fertilidad y resistencia. Debido a que son portadoras de información genética única e importante (López et al., 2001) poseen ventajas indispensables en los sistemas productivos que se desarrollan en estas regiones, cuando se comparan con razas especializadas de carne (Muchenje et al., 2008) que no cuentan con la adaptación necesaria para hacer frente a los ambientes hostiles del trópico sudamericano. La inclusión de razas criollas en programas de mejora y selección por medio de su cruce con razas convencionalmente utilizadas en producción de carne es una forma de incrementar la competitividad del sector cárnico colombiano e impedir la pérdida de este recurso genético. Uno de los principales objetivos del mejoramiento genético en bovinos está relacionado con los atributos de calidad cárnica, que se subdividen en un grupo amplio de cualidades, como son la terneza, la jugosidad (Huff-Lonergan y Lonergan 2005 y 2008) y el flavor. De ellas, la jugosidad estaría estrechamente ligada a la CRA, y es definida como la cantidad de agua que es mantenida en la estructura de la carne al momento cumplir algún proceso tecnológico, como maduración, porcionado, picado, congelación y cocción, sufriendo la menor merma en su peso.

\section{CAmbios en El músculo duRANTE EL POST MÓRTEM (PM)}

Además del contenido, localización y movilidad del agua existen factores antemortem (raza, músculo, estrés pre-sacrificio, el genotipo, dieta, entre otros) y post mórtem (procedimiento de sacrificio, tiempo y temperatura de maduración) que pueden afectar la calidad de la carne (Harper, 1999; Pearce et al., 2011; Ouali et al., 2006). Una vez se realiza el sacrificio del animal se desencadenan una serie de cambios en el músculo hasta su conversión en carne: apoptosis o muerte celular programada, rígor mortis y tenderización.

Al detenerse la circulación sanguínea se inicia la apoptosis al perderse el suministro de nutrientes y oxígeno. Hay una actividad constante de las ATPasas sarcoplasmáticas, causando la caída del nivel de ATP, produciendo simultáneamente fósforo inorgánico que estimula la degradación del glucógeno a ácido láctico. La re-síntesis de ATP por glucólisis anaeróbica es incapaz de mantener el nivel de ATP debido a que es un proceso poco eficaz y a medida que desciende este nivel energético, se forman los complejos de acto-miosina causando la inextensibilidad propia del músculo en rígor mortis (Zhang et al., 2006). En la fase de rígor mortis el músculo se hace inextensible (hallándose en estado de contracción) dado que los filamentos de actina y miosina se sobreponen completamente, formándose muchos enlaces cruzados (Zhang et al., 2006).

Antes de que se alcance el $\mathrm{pH}$ final se inician otros cambios degradativos cuya intensidad afecta la naturaleza y la cantidad de las proteínas y es limitado por la cocción 
(Zhang et al., 2006) denominado como fase de tenderización. La proteólisis contribuye a esta alteración a través de la degradación de proteínas, lo que ha sido observado en músculos mantenidos a temperaturas de refrigeración en un proceso de maduración. Sin embargo, factores físicos como el grado de acortamiento del músculo también contribuyen a la dureza final (Te Pas et al., 2004). El objetivo de este estudio fue establecer el efecto del tipo genético y la maduración sobre la capacidad de retención de agua en carne cruda y cocinada.

\section{MATERIAL Y MÉTODOS}

\section{Material eXPeRIMENTAL}

Fueron evaluados 164 bovinos F1 castrados, provenientes del cruce de hembras de la raza Brahman Blanco (BB) con machos de diferentes razas, obteniendo una población F1 de composición: Limousin-BB: 20; Normanda-BB: 17; Braunvieh-BB: 8; Simmental-BB: 18; Blanco Orejinegro (BON)-BB: 18; Romosinuano-BB: 12; Guzerat-BB: 17; Brahman Rojo (BR)-BB: 10; BB puro: 44 animales, todos mantenidos en dos fincas del sur del Cesar Colombiano. Los individuos F1 fueron alimentados con una dieta a base de los forrajes y sacrificados a un peso promedio de $492 \pm 38,5 \mathrm{~kg}$ y una edad de $30,9 \pm 4,15$ meses en una planta de sacrificio (La Dorada, Caldas, Colombia), mediante procedimientos estándares. Los músculos Longissimus dorsi (LD) y Semitendinosus (ST) fueron extraídos de la canal izquierda a las 24 horas y empacados al vacio. Cada músculo fue dividido en tres porciones (para los diferentes tiempos de maduración 7, 14 y 21 días) y posteriormente cada porción fue dividida en dos piezas (la pieza 1 fue utilizada para la medición de parámetros en carne cruda y la pieza 2 en carne cocinada), las cuales fueron empacadas al vacio y sometidas al proceso de maduración a $4{ }^{\circ} \mathrm{C}$ en un refrigerador industrial. Cumplidos los tiempos de maduración, la pieza 1 fue dividida en tres filetes de 2,54 cm de grosor en los cuales se midió la capacidad de retención de agua en carne cruda (CRAr); la pieza 2 fue sometida a congelación $\left(-20^{\circ} \mathrm{C}\right)$ durante un mes, hasta su procesamiento realizando el fileteado con sierra eléctrica (tres filetes de $2,54 \mathrm{~cm}$ de grosor). Posteriormente los filetes de la pieza 2 fueron descongelados durante 24 horas en refrigeración para la medición de la capacidad de retención de agua en carne cocinada CRAc.

\section{DETERMINACIÓN DE LOS PARÁMETROS DE CALIDAD CÁRNICA}

La CRAr se midió por presión sobre papel filtro (Grau y Hamm, 1953) modificado por Van Oeckel et al. (1999); con una muestra de $0,4 \pm 0,02 \mathrm{~g}$ de carne mediante el texturómetro TA.TX plus (Stable Micro Systems), con una fuerza de $2,2 \mathrm{~kg}$ por 5 minutos y posterior medición de áreas (Zamorano y Gambaruto, 1997). Para medir la CRAc se empleó la metodología sugerida por Bertram et al. (2003) cocinando cada filete en una plancha de doble contacto hasta que el centro geométrico de la pieza cárnica alcanzó los $70^{\circ} \mathrm{C}$, medido mediante termopar.

\section{ANÁLISIS ESTADÍSTICO}

Para el análisis estadístico se utilizó el procedimiento GLM del paquete SAS (SAS, 2008). El modelo aplicado fue el siguiente:

$$
Y_{i j k l m}=\mu+C_{i}+M_{j}+C_{i}^{*} M_{j}+G_{k}+A l(G k)+\varepsilon_{i j k l m}
$$

donde:

$\mathrm{Y}_{\mathrm{ijk} / \mathrm{l}}=$ variable CRA;

$\mu=$ media poblacional;

$\mathrm{C}_{\mathrm{i}}=$ efecto del tipo genético i-ésimo;

$\mathrm{M}_{\mathrm{i}}=$ efecto del tiempo de maduración j-ésimo;

$C_{i}^{*} M_{i}=$ interacción tipo genético*maduración;

$\mathrm{G}_{\mathrm{k}}=$ efecto del grupo contemporáneo k-ésimo (animales de igual procedencia y día de sacrificio);

$\mathrm{Al}(\mathrm{Gk})=$ animal l-ésimo anidado dentro del grupo contemporáneo k-ésimo;

$\varepsilon_{\mathrm{ijkl}}=$ error aleatorio.

De manera independiente, fueron incluidas como covariables la edad y el peso al 


\section{LEAL-GUTIÉRREZ ET AL.}

sacrificio, pero ninguna de las dos se comportó como tal, por lo que no fueron incluidas en el modelo final. Para comprobar la significancia de los factores tipo genético, maduración y su interacción se empleó el anidamiento $\mathrm{Al}$ (Gk) y se aplicaron comparaciones de Tukey-Kramer a los promedios ajustados.

\section{RESULTADOS}

El material experimental correspondió a los 164 primeros animales nacidos, manejados bajo condiciones similares en cada finca, dieta, peso y edad de sacrificio establecido, al igual que las condiciones de sacrificio, manejo de los músculos post mórtem (PM) y metodología para la obtención de los valores de CRAr y CRAc. El modelo presentó varianzas homogéneas, pequeñas y media del error cero, se cumple normalidad del error. Los valores de $\mathrm{R}^{2}$ del modelo para ambos músculos fueron bajos; para CRAr 0,35 en LD y 0,29 en ST y para CRAc, 0,29 en LD y 0,33 en ST, indicando que el modelo explica en estos porcentajes la variación de la CRAr y la CRAc. Los coeficientes de variación fueron para CRAr 0,18 en LD y 0,18 en ST y para CRAc, 0,31 en LD y 0,31 en ST.

\section{Capacidad de ReTENCIÓn DE AGUa EN CARNE CRUDA (CRAR)}

Para el parámetro CRAr en LD, los factores principales, tipo genético, maduración fueron significativos pero su interacción no; para ST se presentó significancia para el factor maduración. En LD, los cruces Braunvieh-BB y Limousin-BB $(36,0 \% \pm 6,21$ y $35,9 \% \pm 8,69$ respectivamente) presentaron diferencias significativas con los cruces BON-BB, Guzerat-BB y BB puro. Para LD se establecieron como ideales periodos cortos de almacenamiento (7-14 días PM). Para el músculo ST, el día 7 de maduración presentó el mejor valor de CRAr de los tres periodos (tabla I).

\section{Capacidad de Retención de agua eN CARNE COCINADA (CRAC)}

Para el parámetro CRAc, el factor principal tipo genético fue significativo en LD, existiendo diferencias significativas entre Limousin-BB $(18,1 \% \pm 6,28)$ y a Braunvieh$\mathrm{BB}$ siendo este último el que presentó el mayor valor en pérdidas por cocinado $(22,9 \% \pm 9,45)$. Para ST ninguno de los factores analizados fue significativo (tabla II).

\section{DISCUSIÓN}

No se encontró una relación entre la edad de los individuos y la característica CRA y algunos resultados con un comportamiento similar son reportados por Gill et al. (2010) y Schönfeldt y Strydom (2011). Estos últimos autores no encontraron un efecto de la edad sobre la CRAr (determinada como pérdidas por goteo) para los músculos ST y Longissimus lumborum cuando utilizaron la edad fisiológica (estructura dental). Sin embargo, es interesante destacar que Sañudo et al. (2004) pudieron establecer que la edad del animal sí afecta las características musculares (dureza, compresión) y es probablemente más notorio en razas más precoces y de bajas tasas de crecimiento diario, cuando se compararon con razas de mayor peso adulto y edad fisiológica más tardía. El comportamiento evidenciado, donde la edad de los animales no correspondió a una covariable de las variables respuesta, CRAr y CRAc, manifiesta que la CRA es determinada principalmente por el agua intracelular y los componentes celulares que la retienen, ya que aproximadamente el $80 \%$ del agua muscular corresponde a aquella retenida por las miofibrillas (Tornberg, 2005). Sin embargo, otras investigaciones (Christensen et al., 2011; Palka y Daun, 1999) destacan el papel desempeñado por el tejido conectivo al cocinar la carne, dada su contracción durante el sometimiento de la pieza cárnica al calor, por lo cual la edad del animal puede influenciar la CRAc debido a la mayor es- 
Tabla I. Significación de factores en la capacidad de retención de agua en carne cruda, de toros cruzados castrados F1 sometida a maduración. (Significative factors of water holding capacity in raw meat of $\mathrm{F} 1$ castrated bulls during aging).

\begin{tabular}{|c|c|c|c|c|c|c|}
\hline \multicolumn{3}{|l|}{ Factor } & LD & \multicolumn{2}{|l|}{ Factor } & ST \\
\hline \multicolumn{3}{|l|}{ Tipo genético } & $<0,0001$ & \multicolumn{2}{|l|}{ Tipo genético } & Ns \\
\hline Maduración & & & 0,0005 & \multicolumn{2}{|l|}{ Maduración } & $<0,0001$ \\
\hline \multicolumn{3}{|c|}{ Tipo genético*Maduración } & Ns & \multicolumn{2}{|c|}{ Tipo genético*Maduración } & Ns \\
\hline & $n$ & $\begin{array}{c}\text { CRAr } \\
\text { Promedio }\end{array}$ & SE & & $\begin{array}{c}\text { CRAr } \\
\text { Promedio }\end{array}$ & SE \\
\hline LD & & & & ST & & \\
\hline $\mathrm{BON}$ & 18 & $31,2^{b}$ & 6,10 & $\mathrm{BON}$ & 29,8 & 5,90 \\
\hline $\mathrm{BB}$ & 44 & $31,3^{b}$ & 6,21 & $\mathrm{BB}$ & 28,4 & 6,34 \\
\hline $\mathrm{BR}$ & 10 & $31,4^{\mathrm{ab}}$ & 7,42 & $\mathrm{BR}$ & 28,0 & 7,72 \\
\hline Braunvieh & 8 & $36,0^{\mathrm{a}}$ & 6,21 & Braunvieh & 28,6 & 4,76 \\
\hline Guzerat & 17 & $30,7^{b}$ & 7,08 & Guzerat & 26,9 & 5,26 \\
\hline Limousin & 20 & $35,9^{a}$ & 8,69 & Limousin & 30,4 & 7,61 \\
\hline Normanda & 17 & $33,3^{\mathrm{ab}}$ & 6,97 & Normanda & 29,8 & 5,25 \\
\hline Romosinuano & 12 & $33,0^{\mathrm{ab}}$ & 5,80 & Romosinuano & 30,1 & 5,32 \\
\hline Simmental & 18 & $31,9^{a b}$ & 7,41 & Simmental & 29,8 & 5,67 \\
\hline 7 días & 164 & $33,7^{x}$ & 7,22 & 7 días & $31,9^{x}$ & 6,24 \\
\hline 14 días & 164 & $33,3^{x}$ & 6,62 & 14 días & $27,7^{y}$ & 5,88 \\
\hline 21 días & 164 & $31,2^{y}$ & 7,12 & 21 días & $27,7^{y}$ & 5,56 \\
\hline
\end{tabular}

Significancia de factores en CRAr; $n=164$; Superíndices diferentes indican significancia $(p<0,05)$; abdentro de cruces. ${ }^{x y}$ dentro de maduración. $\mathrm{BON}=$ Blanco Orejinegro; $\mathrm{BB}=$ Brahaman blano; $\mathrm{BR}=$ Brahman rojo; LD= Longissimus dorsi; $\mathrm{ST}=$ Semitendinosus.

tabilidad que posee este componente a medida que se incrementa la edad del individuo. Adicionalmente otro componente de importancia es establecido por la grasa, dado que el componente graso no retiene agua, pero por otro lado, puede contribuir a incrementar el parámetro CRAc al momento de perderse parte del contenido graso por fusión a altas temperaturas.

\section{LOS FACTORES TIPO GENÉTICO, MADURA- CIÓNYSU INTERACCIÓN}

El factor tipo genético fue significativo para el músculo LD en el parámetro CRAr y en CRAc. Algunos resultados con un comportamiento similar fueron reportados por Zea et al. (2007) en Holstein, Rubia Gallega y su cruce, estableciendo un efecto del tipo genético sobre la CRA (pérdidas por presión en machos) reportándolo como de importancia para estas razas y su cruce. En el análisis realizado por Mariño et al. (2005) hallaron un efecto de la raza sobre el valor de $\mathrm{pH}$ en animales Nelore (Bos indicus) y Holstein (Bos taurus). Sin embargo, en el estudio de Strydom et al. (2000) reportan el efecto de la raza como no significativo sobre parámetros como jugosidad y terneza, utilizando las razas Afrikaner, Nguni, Bonsmara, Santa Gertrudis, Parda Alpina y Pinzgauer. En el estudio de Sañudo et al. (1998) no se encontró efecto alguno del factor raza $(\mathrm{Ru}-$ bia Gallega y Parda Alpina, entre otras) sobre la CRAc al analizar el músculo LD madurado a 7 días, de modo similar al reporte de Zea et al. (2008) cuando evaluaron esta 


\section{LEAL-GUTIÉRREZ ET AL.}

Tabla II. Significación de factores de la capacidad de retención de agua en carne cocinada, de toros cruzados castrados F1 sometida a maduración. (Significative factors of water holding capacity in cooked meat of F1 castrated bulls during aging).

\begin{tabular}{|c|c|c|c|c|c|c|}
\hline \multicolumn{3}{|l|}{ Factor } & LD & \multicolumn{2}{|l|}{ Factor } & ST \\
\hline \multicolumn{3}{|l|}{ Tipo genético } & 0,0365 & \multicolumn{2}{|l|}{ Tipo genético } & ns \\
\hline \multicolumn{3}{|c|}{ Maduración } & ns & \multicolumn{2}{|c|}{ Maduración } & ns \\
\hline \multicolumn{3}{|c|}{ Tipo genético*Maduración } & ns & \multicolumn{2}{|c|}{ Tipo genético*Maduración } & ns \\
\hline & $\mathrm{n}$ & $\begin{array}{c}\text { CRAr } \\
\text { Promedio }\end{array}$ & SE & & $\begin{array}{c}\text { CRAr } \\
\text { Promedio }\end{array}$ & SE \\
\hline LD & & & & ST & & \\
\hline $\mathrm{BON}$ & 18 & $19,4^{\mathrm{ab}}$ & 5,76 & BON & 23,8 & 9,35 \\
\hline $\mathrm{BB}$ & 44 & $20,0^{\mathrm{ab}}$ & 7,36 & BB & 22,7 & 7,96 \\
\hline BR & 10 & $19,3^{\mathrm{ab}}$ & 7,99 & $\mathrm{BR}$ & 24,3 & 8,90 \\
\hline Braunvieh & 8 & $22,9^{a}$ & 9,45 & Braunvieh & 25,9 & 7,18 \\
\hline Guzerat & 17 & $21,4^{\mathrm{ab}}$ & 7,52 & Guzerat & 24,4 & 8,96 \\
\hline Limousin & 20 & $18,1^{\mathrm{b}}$ & 6,28 & Limousin & 23,8 & 8,44 \\
\hline Normando & 17 & $19,9^{a b}$ & 6,10 & Normando & 23,2 & 8,21 \\
\hline Romosinuano & 12 & $22,4^{\mathrm{ab}}$ & 8,45 & Romosinuano & 25,2 & 7,24 \\
\hline Simmental & 18 & $18,5^{\mathrm{ab}}$ & 7,38 & Simmental & 23,8 & 8,76 \\
\hline 7 días & 164 & 20,4 & 6,95 & 7 días & 24,9 & 7,75 \\
\hline 14 días & 164 & 19,4 & 7,40 & 14 días & 24,3 & 7,77 \\
\hline 21 días & 164 & 20,8 & 7,56 & 21 días & 23,2 & 9,69 \\
\hline
\end{tabular}

Significancia de factores en CRAc; $n=164$; abSuperíndices distintos dentro de cruces indican diferencias $(\mathrm{p}<0,05)$. $\mathrm{BON}=$ Blanco Orejinegro; $\mathrm{BB}=\mathrm{Brahman}$ blano; $\mathrm{BR}=$ Brahman rojo; $\mathrm{LD}=$ Longissimus dorsi, $\mathrm{ST}=$ Semitendinosus.

misma característica en machos de las razas Rubia Gallega, Holstein y su cruce. Las diferencias establecidas entre los hallazgos de Zea et al. (2007) y Zea et al. (2008) pueden ser causadas por los objetivos de cada una de las dos investigaciones, la primera adicionalmente al sexo y tipo genético, incluía en el modelo diferentes dietas y en el segundo, los factores evaluados fueron sexo, tipo genético y peso al sacrificio, lo que las hace no comparables entre sí. De lo anterior, se puede deducir que el efecto del tipo genético sobre estas características, está sujeto a los grupos raciales que son evaluados en cada investigación. Estas diferencias en características de calidad han sido encontradas tanto en razas nativas como en razas especializadas de bovinos (Sañudo et al., 1998; Campo et al., 2000; Muchenje et al., 2008; Sañudo et al., 2004; Monsón et al., 2005), lo que está relacionado con las diferencias fisiológicas existentes entre ellas y, por ende, con variaciones en la composición muscular (contenido de proteína, minerales y humedad (Muchenje et al., 2008) lo que puede tener un efecto importante sobre características como la CRA. De forma notoria, en el estudio de Chambaz et al. (2003) pudieron detectar una jerarquización de tipo sensorial para la jugosidad cuando evaluaron el músculo LD a los 14 días PM, así: Angus < Simmental $<$ Charolais $<$ Limousin. Cuando se evaluaron las pérdidas por cocinado para los cruces Simmental-BB y Limousin-BB, se observó una jerarquización inversa. Lo anterior 
está en concordancia con los resultados del presente estudio, en el que el cruce con Limousin fue el que presentó un mejor comportamiento de ambas características, CRAr y CRAc en el músculo LD cuando se comparó con los demás cruces.

Las diferencias raciales establecidas en este estudio son generadas por el efecto de polimorfismos en genes mayores o la suma de pequeños efectos de un grupo muy grande de ellos y su interacción, los cuales presentan diferentes frecuencias alélicas para cada raza, como resultado de la selección a la que han sido sometidas (natural o artificial). En cerdo se han identificado algunos genes mayores con polimorfismos asociados a un efecto importante sobre la característica CRAr, entre los cuales están PRKAG3 y RYR1. En el gen PRKAG3 se identificó la mutación RN-(199V-200Q) presente solamente en la raza Hampshire (Enfält et al., 2006; Lindahl et al., 2004a y b; Bertram et al., 2003) la cual tiene un efecto negativo importante sobre parámetros de textura cárnica. Este efecto fue demostrado en el estudio de Jeremiah et al. (1999), quienes pudieron establecer una jerarquización para el parámetro CRAc en el siguiente sentido: Duroc $<$ Yorkshire < Landrace $<$ Hampshire, siendo la presencia del alelo RN- (199V200Q) determinante sobre el comportamiento de este parámetro de calidad cárnica en la última raza. Cabe destacar el trabajo realizado por Lindahl et al. (2004a) quienes encontraron los mayores valores de pérdidas por goteo (PPG) en el músculo LD de cerdos Hampshire portadores de la mutación RN(199V-200Q) cuando fueron comparados con cerdos normales, ya que en este estudio no se hallaron animales dismutados en la población analizada. Otra mutación que fue identificada en este mismo gen, es la denominada rn* (199I-200R) que tiene un efecto positivo sobre la CRA (Lindahl et al., 2004a y b) y mostró una elevada frecuencia alélica en poblaciones cruzadas de las razas Hampshire y Landrace utilizadas en el estudio de Enfält et al. (2006). Roux et al. (2006) cuando realizaron una caracterización de este mismo gen en bovinos de las razas Limousin, Charolais y Holstein identificaron 21 SNPs y al determinar las diferencias de las frecuencias alélicas por raza para cada mutación encontraron además que algunas mutaciones eran exclusivas de alguna raza, por lo que se podría considerar que estas diferencias genéticas son las responsables de variaciones en las características de calidad cárnica por raza. El segundo gen mayor es RYR, en el que se identificó la mutación R615C en porcinos, la cual incrementa las pérdidas por goteo a 24 horas PM (Otto et al., 2007). Esta mutación ha sido identificada en varias razas de cerdos (Laville et al., 2009; Lahucky et al., 1997; Otto et al., 2007) en las que producía desde alteraciones en la calidad cárnica hasta la muerte durante el transporte.

El factor maduración fue significativo para la CRAr únicamente, indicando que los valores de esta característica presentan una evolución a través de los tres tiempos evaluados, pero al cocinar la carne, los valores de CRAc se hacen estables independientemente del tiempo de maduración de la carne. Cabe destacar que en el presente trabajo se estableció un mejor comportamiento para CRAr a tiempos cortos de maduración (día 7 en LD y a días 7 y 14 en ST), aunque existen varios trabajos que han reportado una tendencia al incremento de la retención de agua a medida que transcurre el tiempo de maduración (Straadt et al., 2007; Zhang et al., 2006; Bond y Warner, 2007; Bee et al., 2007; Monsón et al., 2005; Oliete et al., 2006). Sin embargo, una tendencia similar a la encontrada en este artículo fue reportada por Silva et al. (1999) cuando evaluaron jugosidad. Es necesario destacar que las diferencias anteriormente citadas pueden ser explicadas por las diferentes metodologías utilizadas en los estudios de Straadt et al. (2007) y Zhang et al. (2006) entre otros, ya que la técnica de pérdidas por goteo según el estudio de Van Oeckel et al. (1999) no presenta asociación con el parámetro sensorial de 


\section{LEAL-GUTIÉRREZ ET AL.}

jugosidad. Sin embargo, en el presente estudio se utilizó la metodología de compresión. Para la determinación del parámetro CRAr, que es la única técnica correlacionada con la jugosidad $(0,27$ con $\mathrm{p} \geq 0,01)$ en el trabajo de Van Oeckel et al. (1999), lo que podría explicar el por qué en este estudio se halló una tendencia similar a la reportada en Silva et al. (1999).

Aunque en el caso del parámetro CRAc no se presentó significancia, algunos investigadores reportan un mejor comportamiento para otros caracteres organolépticos de la carne, al incrementar el tiempo de maduración (Slice Shear Force-SSF y compresión, Sañudo et al., 2004 y Monsón et al., 2005). De modo similar, Campo et al. (2000) reportaron que la máxima tenderización (hasta un 99,76\%) de la carne ocurre durante la primera semana de maduración, lo que podría explicar el efecto beneficioso de la proteólisis sobre características como la CRAr y CRAc. En general, en el presente trabajo se pudo establecer que el factor maduración presenta un efecto importante sobre CRAr pero no sobre la CRAc, siendo esta última principalmente afectada por factores involucrados con el procedimiento de cocinado, como el tamaño y la forma del filete y tiempo requerido (Barbanti y Pasquini, 2005), que difícilmente pueden ser estandarizados en un $100 \%$. Lo anterior concuerda con los resultados de otros grupos de investigación que reportan que el período de maduración no tiene efecto sobre la CRAc (Oliete et al., 2006; Straadt et al., 2007).

La interacción tipo genético*maduración no fue significativa para ninguno de los dos músculos evaluados en CRAr ni en CRAc,

\section{BIBLIOGRAFIA}

Anzola, H. 2005. Conservación y utilización de las razas bovinas criollas y colombianas para el desarrollo rural sostenible. Arch Zootec, 54: 141144.

Barbanti, D. and Pasquini, M. 2005. Influence of cooking conditions on cooking loss and tender- estableciendo que no existe un comportamiento diferencial de alguno de los cruces en los tres tiempos de maduración analizados. A diferencia del presente trabajo, esta interacción ha sido reportada por Monsón et al. (2005) para terneza en carne bovina y por Bee et al. (2007) para la CRA en cerdo. Cabe destacar que los estudios de Monsón et al. (2004 y 2005) y Sañudo et al. (2004) concluyen que las diferencias entre razas se hacen menos notorias a medida que se incrementa el tiempo de maduración, principalmente para características asociadas a terneza.

Según la significancia de los factores evaluados, se podría establecer que la evolución de la característica CRAr a lo largo de la maduración presenta un comportamiento complejo en ambos tipos de músculo: LD y $\mathrm{ST}$, ya que poseen diferencias tales como el contenido de colágeno (Morón et al., 2004), diferencias en actividad enzimática, grasa y tipo de fibras (Monsón et al., 2004), características estructurales del músculo y fisiología de la carne (Sañudo et al., 2004), las cuales se hacen más complejas debido a diferencias inherentes del tipo genético. Para la característica CRAc existen algunas diferencias entre los dos músculos analizados indicando que existe una respuesta diferente de cada músculo a este proceso.

\section{CONCLUSIONES}

El tipo genético y el tiempo de maduración de la carne de machos bovinos castrados influyen sobre la CRAr en el músculo LD y el tiempo de maduración para el músculo ST. El tipo genético afecta a la CRAc en el músculo LD.

ness of raw and marinated chicken breast meat. Food Sci Technol-LEB, 38: 895-901.

Bee, G.; Anderson, A.; Lonergan, S. and HuffLonergan, E. 2007. Rate and extent of $\mathrm{pH}$ decline affect proteolysis of cytoskeletal proteins and water-holding capacity in pork. Meat Sci, 


\section{TIPO GENÉTICO, MADURACIÓN Y CAPACIDAD DE RETENCIÓN DE AGUA DE CARNE}

76: 359-365.

Bertram, H.; Andersen, H.; Karlsson, A.; Horn, P.; Hedegaard, J.; Nørgaard, L. and Engelsen, S. 2003. Prediction of technological quality (cooking loss and Napole yield) of pork based on fresh meat characteristics. Meat Sci, 65: 707-712.

Beteta, O. 1997. Las razas autóctonas españolas y su participación en los bovinos criollos iberoamericanos. Razas bovinas creadas en Latinoamérica y el Caribe. Federación Española de Asociaciones de Ganado Selecto. Madrid, España.

Bond, J. and Warner, R. 2007. Ion distribution and protein proteolysis affect water holding capacity of Longissimus thoracis and lumborum in meat of lamb subjected to antemortem exercise. Meat Sci, 75: 406-414.

Campo, M.; Santolaria, P.; Sañudo, C.; Lepetit, J.; Olleta, J.; Panea, B. and Albertí, P. 2000. Assessment of breed type and ageing time effects on beef meat quality using two different texture devices. Meat Sci, 55: 371-378.

Chambaz, A.; Scheeder, M.; Kreuzer, M. and Dufey, P. 2003. Meat quality of Angus, Simmental, Charolais and Limousin steers compared at the same intramuscular fat content. Meat Sci, 63: 491-500.

Christensen, L.; Bertram, H.; Aaslyng, M. and Christensen, M. 2011. Protein denaturation and water-protein interactions as affected by low temperature long time treatment of porcine Longissimus dorsi. Meat Sci, 88: 718-722.

Enfält, A.; Von Seth, G.; Josell, A.; Lindahl, G.; Hedebro, I.; Braunschweig, M.; Andersson, L. and Lundström, K. 2006. Effects of a second mutant allele (V199I) at the PRKAG3 (RN) locus on carcass composition in pigs. Livest Sci, 99: 131-139.

Gill, J.; Bishop, S.; McCorquodale, C.; Williams, J. and Wiener, P. 2010. Associations between single nucleotide polymorphisms in multiple candidate genes and carcass and meat quality traits in a commercial Angus-cross population. Meat Sci, 86: 985-993.

Harper, G. 1999. Trends in skeletal muscle biology and the understanding of toughness in beef. Aust J Agric Res, 50: 1105-1129.

Huff-Lonergan, E. and Lonergan, S. 2005. Mechanisms of water-holding capacity of meat: The role of post mortem biochemical and structural changes. Meat Sci, 71: 194-204.
Huff-Lonergan, E. and Lonergan, S. 2008. Interaction between structure and proteolytic tenderization in beef. Departament of Animal Science. lowa State Universite. Ames, IA 50011, USA. Jeremiah, L.; Gibson, J.; Gibson, L.; Ball, R.; Aker, C. and Fortin, A. 1999. The influence of breed, gender, and PSS (halothane) genotype on meat quality, cooking loss, and palatability of pork. Food Res Int, 32: 59-71.

Lahucky, R.; Christian, L.; Kovaq, L.; Staldert, K. and Bauerova, M. 1997. Meat quality assessed ante- and post mortem by different ryanodine receptor gene status of pigs. Meat Sci, 41:277-285. Laville, E.; Sayd, T.;Terlouw, C.; Blinet, S.; Pinguet, J.; Fillaut, M.; Glenisson, J. and Cherel, P. 2009. Differences in pig muscle proteome according to HAL genotype: Implications for meat quality. J Agri Food Chem, 57: 4913-4923.

Lindahl, G.; Enält, A.; Von Seth, G.; Josell, A.; Hedebro, I.; Andersen, H.; Braunschweig, M.; Andersson, L. and Lundstöm, K. 2004a. A second mutant allele (V199I) at the PRKAG3 (RN) locus-I. Effect on technological meat quality of pork loin. Meat Sci, 66: 609-619.

Lindahl, G.; Enält, A.; Von Seth, G.; Josell, A.; Hedebro, I.; Andersen, H.; Braunschweig, M.; Andersson, L. and Lundstöm, K. 2004b. A second mutant allele (V199I) at the PRKAG3 (RN) locus-II. Effect on colour characteristics of pork loin. Meat Sci, 66: 621-627.

López, A.; Saldarriaga, O.; Arango, A.; Rugeles, M.; Zuluaga, F.; Olivera, M.; Bermúdez, N.; Bedoya, G. y Ossa, J. 2001. Ganado Blanco Orejinegro (BON): Una alternativa para la producción en Colombia. Rev Colom Cienc Pecua, 14: 121-128.

Mariño, G.; Vilca, M. y Ramos, D. 2005. Evaluación del $\mathrm{pH}$ en canales de toros Holstein (Bos taurus) y Nelore (Bos indicus). Rev Investig Vet Perú, 16: 90-95.

Monsón, F.; Sañudo, C. and Sierra, I. 2004. Influence of cattle breed and ageing time on textural meat quality. Meat Sci, 68: 595-602.

Monsón, F.; Sañudo, C. and Sierra, I. 2005. Influence of breed and ageing time on the sensory meat quality and consumer acceptability in intensively reared beef. Meat Sci, 71: 471-479.

Morón, O.; González, N. y Vázquez, F. 2004. Contenido de colágeno y sus fracciones en tres músculos de toretes comerciales. Rev Cient. 


\section{LEAL-GUTIÉRREZ ETAL.}

Universidad de Zulia. 3: 270-273.

Muchenje, V.; Dzama, K.; Chimonyo, M.; Raats, J. and Strydom, P. 2008. Meat quality of Nguni, Bonsmara and Aberdeen Angus steers raised on natural pasture in the Eastern Cape. South Africa. Meat Sci, 79: 20-28.

Oliete, B.; Moreno, T.; Carballo, J.; Monserrat, L. y Sanchez, L. 2006. Estudio de la calidad de carne de ternera de la raza Rubia Gallega a lo largo de la maduración al vacio. Arch Zootec, 55: 3-14.

Otto, G.; Roehe, R.; Looft, H.; Thoelking, L.; Knap, P.; Rothschild, M.; Plastow, G. and Kalm, E. 2007. Associations of DNA markers with meat quality traits in pigs with emphasis on drip loss. Meat Sci, 75: 185-195.

Ouali, A.; Herrera, C.; Coulis, G.; Becila, S.; Boudjellal, A.; Aubry, L. and Sentandreu, M. 2006. Revisiting the conversion of muscle into meat and the underlying mechanisms. Meat Sci, 74: 44-58.

Palka, K. and Daun, H. 1999. Changes in texture, cooking losses, and myofibrillar structure of bovine $M$. semitendinosus during heating. Meat Sci, 51: 237-243.

Pearce, K.; Rosenvold, K.; Andersen, H. and Hopkins, D. 2011. Water distribution and mobility in meat during the conversion of muscle to meat and ageing and the impacts on fresh meat quality attributes. Meat Sci, 89: 111-124.

Renand, G.; Picard, B.; Touraille, C.; Berge, P. and Lepetit, J. 2001. Relationships between muscle characteristics and meat quality traits of young Charolais bulls. Meat Sci, 59: 49-60.

Roux, M.; Nizou, A.; Forestier, L.; Ouali, A.; Levéziel, H. and Amarger, V. 2006. Characterization of the bovine PRKAG3 gene: structure, polymorphism, and alternative transcripts. Mamm Genome, 17: 83-92.

Sánchez, L.; Carballo, J.; Sánchez, B. y Monserrat, L. 2005. Características de la canal y de la carne de machos procedentes del cruce de Rubia Gallega con Nelore. Arch Zootec, 54: 485-489.

Sañudo, C.; Albertí, P.; Campo, M.; Olleta, J. y Panea, B. 1998. Calidad instrumental de la carne de bovino de siete razas españolas. Arch Zootec, 48: 397-402.

Sañudo, C.; Macie, E.; Olleta, J.; Villarroel, M.; Panea, B. and Albertí, P. 2004. The effects of slaughter weight, breed type and ageing time on beef meat quality using two different texture devices. Meat Sci, 66: 925-932.

SAS. 2008. SAS/STAT. User's guide, version 9.2. SAS Institute Inc. Cary, NC. USA.

Schönfeldt, H. and Strydom, P. 2011. Effect of age and cut on cooking loss, juiciness and flavour of South African beef. Meat Sci, 87: 180-190.

Silva, J.; Patarata, L. and Martins, C. 1999. Influence of ultimate $\mathrm{pH}$ on bovine meat tenderness during ageing. Meat Sci, 52: 453-459.

Straadt, I.; Rasmussen, M.; Andersen, H. and Bertram, H. 2007. Aging-induced changes in microstructure and water distribution in fresh and cooked pork in relation to water-holding capacity and cooking loss- A combined confocal laser scanning microscopy (CLSM) and lowfield nuclear magnetic resonance relaxation study. Meat Sci, 75: 687-695.

Strydom, P.; Naude, R.; Smith, M.; Scholtz, M. and Van Wyk, J. 2000. Characterisation of indigenous African cattle breeds in relation to meat quality traits. Meat Sci, 55: 79-88.

Te Pas, M.; Everts, M. and Haagsman, H. 2004. Muscle development of livestock animals. Physiology, genetics and meat quality. CABI Publishing. London.

Tornberg, E. 2005. Effects of heat on meat proteinsImplications on structure and quality of meat products. Meat Sci, 70: 493-508.

Van Oeckel, M.; Warnants, N. and Boucque, V. 1999. Comparison of different methods for measuring water holding capacity and juiciness of pork versus on-line screening methods. Meat Sci, 51: 313-320.

Zamorano, J. and Gambaruto, M. 1997. Contribution to improving the meat water holding capacity test by the filter paper press method. A comparison of three methods for measuring areas. Meat Sci, 46: 129-137.

Zea, J.; Díaz, M. y Carballo, J. 2007. Efecto de la raza, sexo y alimentación en la calidad de la carne de vacuno. Arch Zootec, 56: 737-743.

Zea, J.; Díaz, M. y Carballo, J. 2008. Efecto del peso de sacrificio y la raza en la carne y la grasa de terneros alimentados con ensilados. Arch Zootec, 57: 101-112.

Zhang, W.; Lonergan, S.; Gardner, M. and HuffLonergan, E. 2006. Contribution of post mortem changes of integrin, desmin and $\mu$-calpain to variation in water holding capacity of pork. Meat Sci, 74: 578-585. 